\title{
An Accurate Closed-Form Approximation of the Average Probability of Error over a Log-Normal Fading Channel
}

\author{
Fabien Héliot, Member, IEEE, Mohammad Ghavami, Member, IEEE, \\ and Mohammad Reza Nakhai, Senior Member, IEEE
}

\begin{abstract}
The log-normal probability distribution is commonly used in wireless communications to model the shadowing and more recently the small scale fading for indoor ultra wideband communications. In this paper, an accurate closed-form approximation of the average probability of error over a lognormal fading channel is derived for various constellation types and sizes. This expression can be used to evaluate and compare easily the symbol-error performance of communication systems over a log-normal fading channel.
\end{abstract}

Index Terms-Log-normal distributions, communication channels, probability, error analysis, approximation methods, closedform expression, Lambert $\mathrm{W}$ function.

\section{INTRODUCTION}

H ISTORICALLY, the log-normal distribution has been mainly used to model the effect of shadowing of the signal due to large obstructions [1]. The effect of shadowing on the performance of wireless communication systems has been mostly studied jointly with the effect of small scale fading [2], [3]. Lately, in 2003, the channel modelling subcommittee of the IEEE 802.15.3a working group for wireless personal area networks released a reference Ultra Wide-Band (UWB) indoor channel, known as the IEEE 802.15.3a channel model [4], based on numerous contributions and experimental results [5], [6]. This channel model, designed for UWB communications [7], encompasses the main features of the Saleh-Valenzuela channel model [8], i.e., multipath rays arrive in clusters and their amplitudes follow double-exponential decay, but the lognormal distribution has been found the best match to represent the amplitude distribution of the channel [5].

The average probability of error for different cellular communication systems, where shadowing is involved on its own or mixed with either Rayleigh or Rician fading, has been derived in [2]. The resulting expression is a computable formula of the average probability of error, obtained by using Hermite polynomial approximation [9], that provides only numerical results. Similarly in [3], an exact formula for determining the outage probability of cellular systems over log-normal

Manuscript received December 4, 2006; revised May 14, 2007; accepted September 18, 2007. The associate editor coordinating the review of this paper and approving it for publication was R. Murch.

F. Héliot is with the Centre for Communication Systems Research, University of Surrey, School of Electronics \& Physical Sciences, Guildford GU2 7HX, UK (e-mail: F.Heliot@ Surrey.ac.uk).

M. Ghavami is with the UWB Communications Group, Kings College London, University of London, Division of Engineering, Strand, London WC2R 2LS, UK (e-mail: mohammad.ghavami@kcl.ac.uk).

M. R. Nakhai is with the Centre for Telecommunications Research, Kings College London, University of London, Division of Engineering, Strand, London WC2R 2LS, UK (e-mail: reza.nakhai@kcl.ac.uk).

Digital Object Identifier 10.1109/TWC.2008.061019. shadowed Rician channels has been presented that can be evaluated by using numerical methods.

In this paper, we derive a closed-form approximation of the average probability of error over a log-normal fading channel, with adjustable accuracy, that proves to be highly accurate for a typical mobile communication range of average error probability, i.e., $\overline{P_{e}} \in\left[10^{-6}, 10^{-1}\right]$, and considering various constellation sizes and types. This approximation is designed using simple interpolation techniques, the Lambert's function [10] and other basic mathematical tools. It is given in terms of the Signal-to-Noise-Ratio (SNR) of the system, the mean and the standard deviation of any log-normally distributed Random Variable (RV) and the symbol constellation parameters. Our expression that is presented under a closed-form has been used in [11] to establish the theoretical performance of singleband UWB systems and to derive code design criteria for multiple-antennae single-band UWB systems operating over the IEEE 802.15.3a channel. It can also be useful to evaluate the symbol-error performance of communication systems over log-normal fading channels or to get an intuitive understanding of the behaviour of this performance at high SNRs.

\section{SYSTEM MODEL}

In a mobile radio environment, shadowing is usually modelled as a multiplicative and slowly time varying random process. The received signal $r(t)$ can be expressed in presence of noise as follows [1]

$$
r(t)=\sqrt{\epsilon_{s}} s(t) h(t)+n(t),
$$

where $\epsilon_{s}$ is the transmitted symbol energy, $s(t)$ is the transmitted signal, $h(t)$ is the random process that characterizes the shadowing effect, and $n(t)$ is an additive zero-mean Gaussian noise with variance $N_{0} / 2$. At any instant, the shadowing is considered as log-normally distributed. Moreover, for a slow-varying environment, $h(t)$ can be considered constant such that $h(t)=\beta$ is a log-normally distributed RV. As far as UWB communication systems are concerned, the channel model released by the IEEE for indoor communications in [4], namely the IEEE 802.15.3a channel model, exhibits a log-normal distribution of its multipath attenuation factor. In that case, the received signal $r(t)$ can be expressed as in (1) with $h(t)=\sum_{k} G_{k} \delta\left(t-\tau_{k}\right)$, as shown in [12] and [13], where $G_{k}$ is the multipath attenuation factor, $\tau_{k}$ is its corresponding time delay, and $\delta(t)$ is an impulse signal. Any multipath attenuation factor $G_{k}$ is such that $G_{k}=\beta_{k} e^{j \theta_{k}}$ with $\beta_{k}$ being a log-normally distributed RV and $\theta_{k} \in\{0, \pi\}$ [4]. Assuming that $s(t)$ is a signal modulated with one of the following common constellations; a binary antipodal, a 
binary orthogonal, an $M$-ary Pulse Amplitude Modulation (PAM), an $M$-ary Phase Shift Keying (PSK) modulation, or a rectangular $M$-ary Quadrature Amplitude Modulation (QAM); then the symbol-error probability obtained by using maximum likelihood detection for these constellations is given by [1]

$$
P_{e}=q(\alpha)=\zeta_{1} Q\left(\sqrt{\zeta_{0} \gamma_{s} \alpha}\right),
$$

where $Q(x)=\frac{1}{\sqrt{2 \pi}} \int_{x}^{\infty} e^{-t^{2} / 2} d t, \zeta_{0}$ and $\zeta_{1}$ are coefficients related to the constellation shape [14], $\gamma_{s}=\epsilon_{s} / N_{0}$ is the SNR per symbol, and $\alpha$ is a RV. In the shadowing case $\alpha=\beta^{2}$ and in the UWB case, as shown in [12] and [13], $\alpha \approx \sum_{k} \beta_{k}^{2}$. Moreover, since $\beta$ is log-normally distributed, $\beta^{2}$ is log-normally distributed as well, and then using the results in [15] $\sum_{k} \beta_{k}^{2}$ can be considered as a log-normally distributed RV [13]. Therefore, in both cases $\alpha$ is a lognormally distributed RV. Averaging (2) over $\alpha$, we obtain an expression for the Average Symbol-Error Probability (ASEP) as

$$
\overline{P_{e}}=\int_{0}^{+\infty} p(\alpha) q(\alpha) d \alpha,
$$

where $p(\alpha)$ is the pdf of a $\left(m_{\alpha}, \sigma_{\alpha}\right)$-log-normally-distributed $\mathrm{RV} \alpha$ given by

$$
p(\alpha)=\frac{1}{\alpha \sqrt{2 \pi} \sigma_{\alpha}} \exp \left(-\frac{1}{2}\left(\frac{\ln (\alpha)-m_{\alpha}}{\sigma_{\alpha}}\right)^{2}\right),
$$

with $m_{a}$ and $\sigma_{a}$ being the mean and standard deviation of the log-normally distributed $\mathrm{RV} \alpha$, respectively. Usually in wireless communications, parameters $\left(m_{y}, \sigma_{y}\right)$ are introduced to characterize a log-normal distribution, where $m_{y}=C m_{\alpha}$, $\sigma_{y}=C \sigma_{\alpha}$ and $C=10 / \ln (10)$ [15]. The parameter $\sigma_{y}$, known as the $\mathrm{dB}$ spread, is usually between 6 and $12 \mathrm{~dB}$ for most of the wireless communication systems [15], and between 2 and $5 \mathrm{~dB}$ for the UWB systems [4]. The function $Q(x)$ can be tightly-approximated or closely-upper bounded by other simple and integrable functions as it has been shown in [2] and [16]. For instance, in [16], $Q(x)$ is said to be upper bounded by

$$
\sum_{i=1}^{I} c_{i} e^{-\frac{b_{i}}{2} x^{2}},
$$

where $c_{i}=a_{i} / 2, a_{i}>0$ and $b_{i} \geq 1$ are defined in [16]. The expression in (5) tends to be equal to $Q(x)$ as $I$ increases. Moreover, for $I=1, c_{1}=b_{1}=1, Q(x) \leq e^{-x^{2} / 2}$ is the Chernoff bound on $Q(x)$. Therefore, using (5) in (2), then (2) and (4) in (3), the ASEP can be re-expressed as

$$
\begin{aligned}
\overline{P_{e}} \simeq & \sum_{i=1}^{I} \int_{0}^{+\infty} \frac{\zeta_{1} c_{i}}{\alpha \sqrt{2 \pi} \sigma_{\alpha}} \times \\
& \exp \left(-\frac{1}{2}\left(\zeta_{0} b_{i} \gamma_{s} \alpha+\left(\frac{\ln (\alpha)-m_{\alpha}}{\sigma_{\alpha}}\right)^{2}\right)\right) d \alpha,
\end{aligned}
$$

for a large value of $I$. Then, using the change of variables $x=\left(\frac{\ln (\alpha)-m_{\alpha}}{\sqrt{2} \sigma_{\alpha}}\right)$ in $(6), \overline{P_{e}}$ can be rewritten in a compact form as

$\overline{P_{e}} \simeq \sum_{i=1}^{I} \frac{\zeta_{1} c_{i}}{\sqrt{\pi}} \int_{-\infty}^{+\infty} e^{-\nu_{i} e^{\frac{x}{k}}} e^{-x^{2}} d x=\sum_{i=1}^{I} \frac{\zeta_{1} c_{i}}{\sqrt{\pi}} \int_{-\infty}^{+\infty} f(x) d x$ where $\nu_{i}=\frac{\zeta_{0} b_{i}}{2} \gamma_{s} e^{m_{\alpha}} \geq 0, k=\frac{1}{\sqrt{2} \sigma_{\alpha}} \geq 0$, and $f(x)$ is expressed as follows

$$
f(x)=e^{-\nu_{i} e^{-\frac{x}{k}}} e^{-x^{2}}, x \in \mathbb{R} .
$$

\section{A Tight Approximation of the InTEgRal of $f(x)$}

In this section, knowing that $f(x)$ does not have a closedform integral, we use linear interpolation to design an analytically integrable function $g(x)$, which tightly approximates $f(x)$ over its domain.

The function $f(x)$ in (8) is always a positive and a differentiable function of $x$ and $\lim _{x \rightarrow-\infty} f(x)=\lim _{x \rightarrow+\infty} f(x)=0$. Differentiating $f(x)$ with respect to $x$, we obtain

$$
\frac{d f(x)}{d x}=e^{-\left(\nu_{i} e^{-\frac{x}{k}}+\frac{x}{k}+x^{2}\right)}\left[\frac{\nu_{i}}{k}-2 x e^{\frac{x}{k}}\right] .
$$

The first term of this expression is clearly always positive. Let $\widehat{x_{\mathrm{f}}}$ be a solution of the equation $\frac{d f(x)}{d x}=0$. Then, $\frac{d f(x)}{d x} \geq 0$ over $\left(-\infty, \widehat{x_{\mathrm{f}}}\right]$, and $\frac{d f(x)}{d x} \leq 0$ over $\left[\widehat{x}_{\mathrm{f}},+\infty\right)$. This implies that $f(x)$ increases over $\left(-\infty, \widehat{x_{\mathrm{f}}}\right]$ and decreases over $\left[\widehat{x}_{\mathrm{f}},+\infty\right)$. Therefore, $f(x)$ has a unique maximum, which occurs at $x=$ $\widehat{x_{\mathrm{f}}}$, that can be obtained as follows

$$
\frac{d f\left(x=\widehat{x_{\mathrm{f}}}\right)}{d x}=0 \Rightarrow \frac{\nu_{i}}{2 k^{2}}=\frac{\widehat{x_{\mathrm{f}}}}{k} e^{\widehat{x_{\mathrm{f}}}} .
$$

The Lambert $W$ function is the inverse function of $f(w)=$ $w e^{w}$ where $e^{w}$ is the natural exponential function and $w$ is any complex number and thus, the Lambert $W$ function satisfies $W(z) e^{W(z)}=z, z \in \mathbb{C}[10]$. Moreover, the real branch of the Lambert function denoted $W_{0}$ is such that $W_{0}: \mathbb{R}_{+} \rightarrow \mathbb{R}_{+}$. Then, in (10), we set $W_{0}(z)=\frac{\widehat{x}_{\mathrm{f}}}{k}, z=\frac{\nu_{i}}{2 k^{2}}$ and obtain [17]

$$
\widehat{x_{\mathrm{f}}}=k W_{0}\left(\frac{\nu_{i}}{2 k^{2}}\right) \text {. }
$$

Since $\nu_{i} \in \mathbb{R}_{+}, k \in \mathbb{R}_{+}$, and $W_{0}: \mathbb{R}_{+} \rightarrow \mathbb{R}_{+}$, therefore $\widehat{x_{\mathrm{f}}} \in \mathbb{R}_{+}$.

For any interval $\left[x_{n}, x_{n+1}\right], x_{n}, x_{n+1} \in \mathbb{R}$, there exists, $a_{n} \geq 0, b_{n} \leq 0$ verifying the inequality

$$
x^{2} \geq a_{n}|x|+b_{n},
$$

and chosen such that $x^{2}=a_{n}|x|+b_{n}$ for $x=\frac{x_{n+1}+x_{n}}{2}$. Inserting the result (12) in (8) and defining the interval $\left[x_{n}, x_{n+1}\right]$ to be narrow, i.e., $\left(x_{n+1}-x_{n}\right) \ll 1$, we obtain a very tight upper-bound of $f(x)$ over each interval, as follows

$$
g_{n}(x)=e^{-\nu_{i} e^{-\frac{x}{k}}} e^{-\left(a_{n}|x|+b_{n}\right)} .
$$

Then, $g(x)$ is defined using $g_{n}(x)$ as

$$
g(x)=\sum_{n=-L N}^{L N} g_{n}(x),
$$

such that for any interval $\left[x_{n}, x_{n+1}\right], g_{n-1}\left(x_{n}\right)=g_{n}\left(x_{n}\right)$ in order to ensure that $g(x)$ is a continuous function over its domain. Therefore, if $\left[x_{n}, x_{n+1}\right]$ is narrow enough, then $g(x)$ tightly upper-bounds $f(x)$, and thus as $f(x)$, is a positive function of $x$ with a unique maximum. Let us denote the maximum of $g(x), \widehat{x_{\mathrm{g}}}$, and assume that this maximum occurs for a $x$ belonging to one of the $2 L N$ intervals $\left[x_{n}, x_{n+1}\right]$ composing the domain of $g(x)$. For instance, let the interval 
$\left[x_{0}, x_{1}\right]$ contains $\widehat{x_{\mathrm{g}}}$, then using the first derivative of $g_{0}(x)$ defined as

$$
\frac{d g_{0}(x)}{d x}=e^{-\left(\nu_{i} e^{-\frac{x}{k}}+\frac{x}{k}+a_{0}|x|+b_{0}\right)}\left[\frac{\nu_{i}}{k}-\frac{a_{0} x}{|x|} e^{\frac{x}{k}}\right]=0,
$$

we can solve $\frac{d g_{0}\left(x=\widehat{x_{\mathrm{g}}}\right)}{d x}=0$ and obtain the value of $\widehat{x_{\mathrm{g}}}$. For $\widehat{x_{\mathrm{g}}}<0,\left|\widehat{x_{\mathrm{g}}}\right|=-\widehat{x_{\mathrm{g}}}$ and it implies with (15) that $\frac{\nu_{i}}{k}=-a_{0} e^{\frac{\widehat{x_{\mathrm{g}}}}{k}}$. This equation has no solution in $\mathbb{R}$ since $\nu_{i}, k, a_{0}$ and $e^{\widehat{x_{g}}}$ are all positive numbers, thus $\widehat{x_{\mathrm{g}}}$ is positive. Then, using (15) $\widehat{x_{\mathrm{g}}}$ can be expressed as

$$
\widehat{x_{\mathrm{g}}}=k \ln \left(\nu_{i} / k a_{0}\right) .
$$

At this point using relation (12), we propose rules to design the intervals $\left[x_{n}, x_{n+1}\right]$ and the parameters $a_{n}$ and $b_{n}$. Knowing that both functions $f(x)$ and $g(x)$ are positive with a single positive maximum, we set both maxima to occur at the same abscissa value such that $\widehat{x_{\mathrm{f}}}=\widehat{x_{\mathrm{g}}}$. Consequently, using the definition of $\widehat{x_{\mathrm{f}}}$ and $\widehat{x_{\mathrm{g}}}$ given in (11) and (16), respectively, it implies $k W_{0}\left(\nu_{i} / 2 k^{2}\right)=k \ln \left(\nu_{i} / k a_{0}\right) \Leftrightarrow \exp \left(W_{0}\left(\nu_{i} / 2 k^{2}\right)\right)=$ $\nu_{i} / k a_{0} \Leftrightarrow\left(a_{0} / 2 k\right) \exp \left(W_{0}\left(\nu_{i} / 2 k^{2}\right)\right)=\nu_{i} / 2 k^{2} \geq 0$, equivalently with the definition of the Lambert function $\left(a_{0} / 2 k\right) \exp \left(W_{0}\left(\nu_{i} / 2 k^{2}\right)\right)=W_{0}\left(\nu_{i} / 2 k^{2}\right) \exp \left(W_{0}\left(\nu_{i} / 2 k^{2}\right)\right)$ and finally gives

$$
a_{0}=2 k W_{0}\left(\frac{\nu_{i}}{2 k^{2}}\right)=2 \widehat{x_{\mathrm{f}}} .
$$

Moreover, we set both maxima to occur at the same ordinate value such that $f(x)=g_{0}(x)$ for $x=\widehat{x_{\mathrm{f}}}=\widehat{x_{\mathrm{g}}}$. Consequently, using the definition of $f(x)$ in (8) and $g_{n}(x)$ in (13) with $n=$ 0 , for $x=\widehat{x_{\mathrm{f}}}$, it comes $\exp \left(-{\widehat{x_{\mathrm{f}}}}^{2}\right)=\exp \left(-\left(a_{0}\left|\widehat{x}_{\mathrm{f}}\right|+b_{0}\right)\right) \Leftrightarrow$ ${\widehat{x_{\mathrm{f}}}}^{2}=a_{0}\left|{\widehat{x_{\mathrm{f}}}}\right|+b_{0} \Leftrightarrow{\widehat{x_{\mathrm{f}}}}^{2}=2{\widehat{x_{\mathrm{f}}}}^{2}+b_{0}$, and thus implies $b_{0}=-\widehat{x}_{\mathrm{f}}^{2}$. Finally, over the interval $\left[x_{0}, x_{1}\right], x_{0}$ and $x_{1}$ must satisfy simultaneously the inequality in (12), the equality $x^{2}=a_{0}|x|+b_{0}$ for $x=\frac{x_{1}+x_{0}}{2}$ and $\left(x_{1}-x_{0}\right) \ll 1$. Thus, we propose $x_{1}=\widehat{x_{\mathrm{f}}}\left(1+\frac{1}{2 N}\right), x_{0}=\widehat{x_{\mathrm{f}}}\left(1-\frac{1}{2 N}\right)$, and the length of $\left[x_{0}, x_{1}\right]$ to be equal to $\widehat{x_{\mathrm{f}}} / N$, with $N \gg 1$. Similarly for the next interval of length $\widehat{x_{\mathrm{f}}} / N$ adjacent to $\left[x_{0}, x_{1}\right]$, i.e., $\left[x_{1}, x_{2}\right]$, using $g_{0}\left(x_{1}\right)=g_{1}\left(x_{1}\right)$, i.e., the continuity of $g(x)$ is maintained at the border of any interval, and $f\left(\frac{x_{2}+x_{1}}{2}\right)=$ $g_{1}\left(\frac{x_{2}+x_{1}}{2}\right)$, i.e., $x^{2}=a_{1}|x|+b_{1}$ for $x=\frac{x_{2}+x_{1}}{2}$, the following equations can be obtained

$\left\{\begin{array}{l}g_{0}\left(x_{1}\right)=g_{1}\left(x_{1}\right) \Rightarrow{\widehat{x_{\mathrm{f}}}}^{2}(1+1 / N)=a_{1} \widehat{x_{\mathrm{f}}}(1+1 / 2 N)+b_{1}, \\ f(x)=g_{1}(x) \Rightarrow{\widehat{x_{\mathrm{f}}}}^{2}(1+1 / N)^{2}=a_{1} \widehat{x_{\mathrm{f}}}(1+1 / N)+b_{1} .\end{array}\right.$

Solving (18) in terms of $a_{1}$ and $b_{1}$, one achieves after simplification $a_{1}=2 \widehat{x_{\mathrm{f}}}(1+1 / N)$ and $b_{1}=-{\widehat{x_{\mathrm{f}}}}^{2}(1+1 / N)^{2}$. Using the same approach on any other interval $\left[x_{n}, x_{n+1}\right]$, we obtain $a_{n}, b_{n}, x_{n}$, as follows,

$$
\begin{cases}a_{n}=2 \widehat{x_{\mathrm{f}}}(1+n / N) & , n \in\{-N, L N\} \\ a_{n}=-2 \widehat{x_{\mathrm{f}}}(1+n / N) & , n \in\{-L N,-N-1\}, \\ b_{n}=-\widehat{x}_{\mathrm{f}}^{2}(1+n / N)^{2} & , n \in\{-L N, L N\} \\ x_{n}=\widehat{x_{\mathrm{f}}}(1+(2 n-1) / 2 N) & , n \in\{-L N, L N\}\end{cases}
$$

The value of $x$ depends on $n$ as follows,

- if $n \geq-N$, then $x \geq 0$ and $a_{n}|x|=a_{n} x=$ $2 \widehat{x_{\mathrm{f}}}(1+n / N) x \geq 0$.

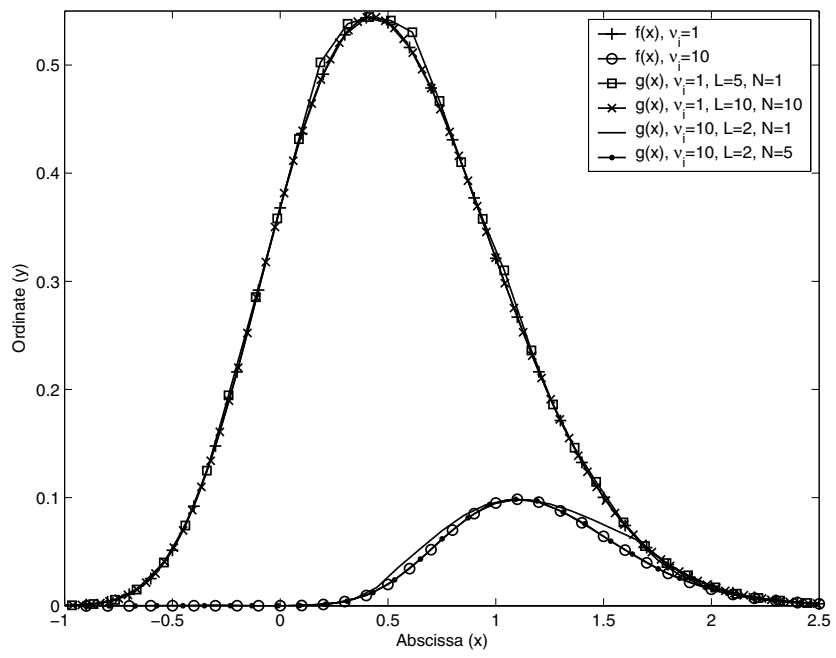

Fig. 1. Comparison of the curves of $f(x)$ and $g(x)$ for $k=0.5$ and various values of $\nu_{i}, L$, and $N$.

- if $n<-N$, then $x<0$ and $a_{n}|x|=-a_{n} x=$ $2 \widehat{x_{\mathrm{f}}}(1+n / N) x>0$.

Therefore, for any $n \in\{-L N, L N\}$ and $x \in$ $\left[x_{-L N}, x_{L N+1}\right], a_{n}|x|=2 \widehat{x_{\mathrm{f}}}(1+n / N) x \geq 0$. Inserting $a_{n}|x|=2 \widehat{x}_{\mathrm{f}}(1+n / N) x$ and $b_{n}=-{\widehat{x_{\mathrm{f}}}}^{2}(1+n / N)^{2}$ in (13), we can rewrite $g_{n}(x)$ over any interval $\left[\widehat{x_{\mathrm{f}}}\left(1+\frac{2 n-1}{2 N}\right), \widehat{x_{\mathrm{f}}}\left(1+\frac{2 n+1}{2 N}\right)\right]$ as follows

$$
g_{n}(x)=e^{-\nu_{i} e^{-\frac{x}{k}}} e^{-\widehat{x}_{\mathrm{f}}\left(1+\frac{n}{N}\right)\left[2 x-\widehat{x}_{\mathrm{f}}\left(1+\frac{n}{N}\right)\right],}
$$

$\forall n \in\{-L N, L N\}, x \in \mathbb{R}$, and verify $f(x) \simeq g(x)$ for a large value of $L N$, as shown in Fig. 1 for $k=0.5$ and different values of $\nu_{i}, L$ and $N$. For instance in the case of $L=10$ and $N=10, f(x)$ and $g(x)$ differs on average by less than $0.001 \%$. Next, according to the property of additivity with respect to interval of integration [18], and using $f(x) \simeq g(x)$, (14), (19), and (20), one can write $F(x)$ as follows

$$
\begin{aligned}
F(x) & =\int_{-\infty}^{+\infty} f(x) d x \simeq \sum_{n=-L N}^{L N} \int_{\widehat{x_{\mathrm{f}}}\left(1+\frac{2 n-1}{2 N}\right)}^{\widehat{x_{\mathrm{f}}}\left(1+\frac{2 n+1}{2 N}\right)} e^{-\nu_{i} e^{-\frac{x}{k}}} \\
& \times e^{-\widehat{x_{\mathrm{f}}}\left(1+\frac{n}{N}\right)\left[2 x-\widehat{x_{\mathrm{f}}}\left(1+\frac{n}{N}\right)\right]} d x .
\end{aligned}
$$

Using the change of variable $t_{i}=\nu_{i} e^{-\frac{x}{k}}$ in (21), this equation can be re-expressed as, [17],

$$
\begin{aligned}
& F(x) \simeq k \sum_{n=-L N}^{L N} \nu_{i}^{-2 k \widehat{x_{\mathrm{f}}}\left(1+\frac{n}{N}\right)} e^{\left(\widehat{x_{\mathrm{f}}}\left(1+\frac{n}{N}\right)\right)^{2}}
\end{aligned}
$$

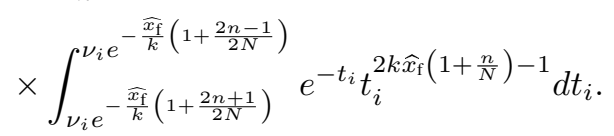

Defining a dummy variable $u_{i}=2 k \widehat{x_{\mathrm{f}}}=$ $W_{0}\left(\frac{\zeta_{0} b_{i}}{2} \gamma_{b} e^{m_{\alpha}} \sigma_{\alpha}^{2}\right) / \sigma_{\alpha}^{2}$, one can simplify $F(x)$ as, [17],

$$
\begin{aligned}
& F(x) \simeq \frac{1}{\sqrt{2} \sigma_{\alpha}} \sum_{n=-L N}^{L N} e^{-u_{i}\left(1+\frac{n}{N}\right)\left[\ln \left(u_{i}\right)+u_{i} \sigma_{\alpha}^{2}\left(\frac{N-n}{2 N}\right)\right]} \\
& \times \int_{u_{i} e^{-\sigma_{\alpha}^{2}\left(\frac{2 n+1}{2 N}\right) u_{i}}}^{u_{i} e^{-\sigma_{\alpha}^{2}\left(\frac{2 n-1}{2 N}\right) u_{i}}} e^{-t_{i}} t_{i}^{u_{i}\left(1+\frac{n}{N}\right)-1} d t_{i},
\end{aligned}
$$




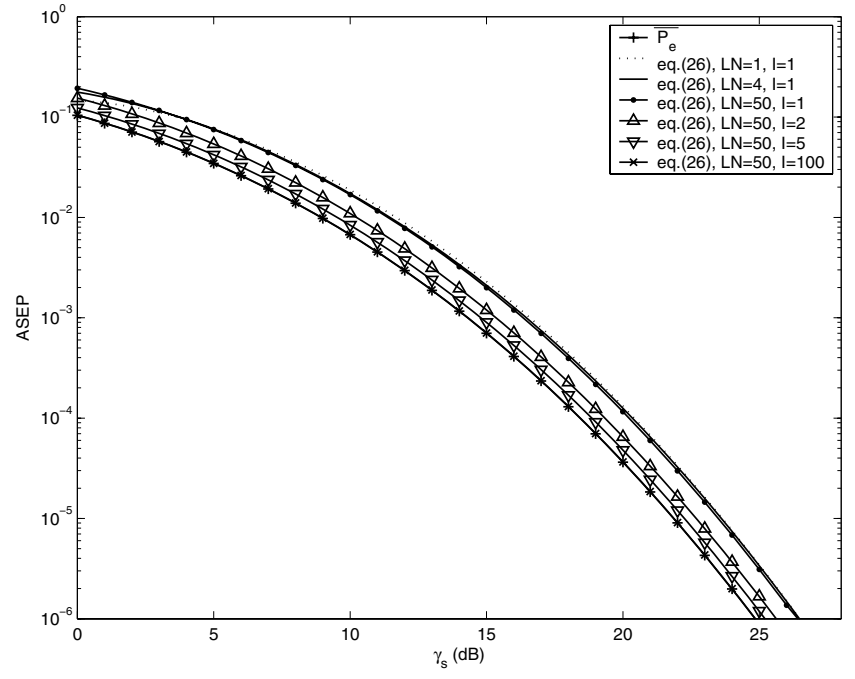

Fig. 2. ASEP vs. SNR per symbol for a binary antipodal constellation, $\sigma_{y}=6 \mathrm{~dB}$, and various values of $L N$ and $I$ using $\widetilde{P_{e}}$, i.e., eq. (26), and $\overline{P_{e}}$ relations.

where the integral part in (23) can be obtained as follows

$$
\begin{gathered}
G\left(u_{i}, N, n\right)=\int_{u_{i} e^{-\sigma_{\alpha}^{2}\left(\frac{2 n+1}{2 N}\right) u_{i}} u^{-\sigma_{\alpha}^{2}\left(\frac{2 n-1}{2 N}\right) u_{i}} e^{-t_{i}} t_{i}^{u_{i}\left(1+\frac{n}{N}\right)-1} d t_{i}}=\left\{\begin{array}{l}
\lambda\left(-u_{i}(1+n / N), U_{i, n, N}^{+}\right)-\lambda\left(-u_{i}(1+n / N),\right. \\
\left.U_{i, n, N}^{-}\right), \text {for } n \in\{-L N,-N-1\}, \\
E_{1}\left(U_{i, n, N}^{+}\right)-E_{1}\left(U_{i, n, N}^{-}\right), \text {for } n=-N, \\
\gamma\left(u_{i}(1+n / N), U_{i, n, N}^{-}\right)-\gamma\left(u_{i}(1+n / N), U_{i, n, N}^{+}\right), \\
\text {for } n \in\{-N+1, L N\} .
\end{array}\right.
\end{gathered}
$$

In (24), $U_{i, n, N}^{+}=u_{i} e^{-\sigma_{\alpha}^{2}\left(\frac{2 n+1}{2 N}\right) u_{i}}, U_{i, n, N}^{-}=u_{i} e^{-\sigma_{\alpha}^{2}\left(\frac{2 n-1}{2 N}\right) u_{i}}$, $\gamma(c, d)=\int_{0}^{d} e^{-t} t^{c-1} d t$ denotes the incomplete Gamma function, $E_{1}(x)=\int_{x}^{+\infty} e^{-t} / t d t$ is the Exponential integral function, and $\lambda(c, d)=\int_{d}^{+\infty} e^{-t} t^{-c-1} d t$, with $c, d \in \mathbb{R}_{+}$ excluding zero. Hence, $F(x)$ is tightly approximated as

$$
\begin{aligned}
F(x) & \simeq \frac{1}{\sqrt{2} \sigma_{\alpha}} \sum_{n=-L N}^{L N} G\left(u_{i}, N, n\right) \\
& \times e^{-u_{i}\left(\frac{N+n}{N}\right)\left[\ln \left(u_{i}\right)+u_{i} \sigma_{\alpha}^{2}\left(\frac{N-n}{2 N}\right)\right] .}
\end{aligned}
$$

Finally, by inserting (25) in (7), we derive an approximation of the APSE as

$$
\begin{aligned}
\overline{P_{e}} \simeq \widetilde{P_{e}} & =\sum_{i=1}^{I} \frac{\zeta_{1} c_{i}}{\sqrt{2 \pi} \sigma_{\alpha}} \sum_{n=-L N}^{L N} G\left(u_{i}, N, n\right) \\
& \times e^{-u_{i}\left(\frac{N+n}{N}\right)\left[\ln \left(u_{i}\right)+u_{i} \sigma_{\alpha}^{2}\left(\frac{N-n}{2 N}\right)\right],}
\end{aligned}
$$

i.e., in terms of known mathematical functions, except for $\lambda(c, d)=\int_{d}^{+\infty} e^{-t} t^{-c-1} d t$.

\section{Numerical ANALysis AND IntUitive SimplificATION METHOD FOR $\overline{P_{e}}$}

In Fig. 2, we plot $\overline{P_{e}}$ and $\widetilde{\bar{P}_{e}}$ against $\gamma_{s}(\mathrm{~dB})$, and compare them considering a binary antipodal constellation, i.e., $\left(\zeta_{0}=\right.$ $\left.2, \zeta_{1}=1\right)$, a dB spread $\sigma_{y}=6 \mathrm{~dB}, m_{y}=0 \mathrm{~dB}$, various values of $I$ and different values of $L N$. As expected, the results first show that the larger $I, N, L$ are, the better the approximation is. The results also indicate that if $I$ decreases then the approximation becomes looser, nevertheless $\widetilde{\overline{P_{e}}}$ keeps a nearly parallel feature with the curve of $\overline{P_{e}}$ for large SNR per symbol values. This parallel feature is observed for any value of $I$. For $I=1, c_{1}=1 / 2$ and $b_{1}=1,[16]$, (26) can be re-expressed as

$$
\widetilde{P_{e}}=\frac{\zeta_{1}}{2 \sqrt{2 \pi} \sigma_{\alpha}} \sum_{n=-L N}^{L N} G(u, N, n) e^{-u\left(\ln (u)+\sigma_{\alpha}^{2} u / 2\right)},
$$

where $u=W_{0}\left(\frac{\zeta_{0}}{2} \gamma_{b} e^{m_{\alpha}} \sigma_{\alpha}^{2}\right) / \sigma_{\alpha}^{2}$. Moreover, for a fixed $I$ value, the values of $L N$ affect the curvature of $\widetilde{\overline{P_{e}}}$ at low SNRs. Notice that the same results are obtained for other constellations, different values of $\sigma_{y}$ and $m_{y}$. Following these observations, it becomes clear that at low SNRs, the slope of $\widetilde{\overline{P_{e}}}$ is predominantly determined by the multiplicative term $G(u, N, n)$ in (27), and that at high SNRs, i.e., $u \gg 1$, the slope of $\overline{P_{e}}$ is predominantly determined by the exponential term in (27). Using this intuition, we modify $\widetilde{P_{e}}$ in (26), which is not convenient to use and is not actually a proper closedform expression, and introduce a new function $\overline{P_{e}^{\prime}}$ based on (27) to closely approximate $\overline{P_{e}}$ as follows

$$
\overline{P_{e}} \simeq \overline{P_{e}^{\prime}}=\frac{\zeta_{1}}{\sqrt{2 \pi} \sigma_{\alpha}} \phi(u) e^{-u\left(\ln (u)+\sigma_{\alpha}^{2} u / 2\right)},
$$

where

$$
\phi(u)=\eta_{0} e^{u \ln (u)-\eta_{1} u^{\eta_{2}}}
$$

has been found to be an excellent choice of a parametric function, with $\eta_{0}, \eta_{1}, \eta_{2} \in \mathbb{R}$. Notice that, contrary to $\widetilde{\overline{P_{e}}}$ in (26), $\overline{P_{e}^{\prime}}$ is a closed-form expression given in terms of the Lambert, logarithmic and exponential functions.

\section{A Highly Accurate Closed-Form Approximation OF THE ASEP}

The tightness of our closed-form approximation of the ASEP proposed in (28) can be adjusted using the parameters $\eta_{0}, \eta_{1}$ and $\eta_{2}$. Our method to find the best approximation of $\overline{P_{e}}$ by $\overline{P_{e}^{\prime}}$ between any two probability of errors $P_{a}$ and $P_{b}$ relies on the following criterion

$$
\begin{aligned}
\frac{1}{N} \sum_{n=0}^{N-1} & \mid \bar{P}_{e}^{-1}\left(10^{\Delta n\left(n+\frac{1}{2}\right)} P_{a}, \sigma_{y}\right) \\
& -{\overline{P_{e}^{\prime}}}^{-1}\left(10^{\Delta n\left(n+\frac{1}{2}\right)} P_{a}, \sigma_{y}, \eta_{0}, \eta_{1}, \eta_{2}\right) \mid<\varepsilon_{0} .
\end{aligned}
$$

In (30), ${\overline{P_{e}}}^{-1}($.$) and {\overline{P_{e}^{\prime}}}^{-1}($.$) are the inverse functions of \overline{P_{e}}$ and $\overline{P_{e}^{\prime}}$, respectively, $\Delta n=\left(\log _{10}\left(P_{b}\right)-\log _{10}\left(P_{a}\right)\right) / N, N$ is the number of discrete samples in the interval $\left(P_{a}, P_{b}\right)$, and $\varepsilon_{0} \ll 1$. Using this criterion, we have obtained $\eta_{0}, \eta_{1}$, and $\eta_{2}$ for $\sigma_{y} \in[2,12]$ with increments of $0.5 \mathrm{~dB}$ for $\sigma_{y}$ values, $P_{a}=10^{-6}, P_{b}=10^{-1}, N=1000, \varepsilon_{0}=0.01 \mathrm{~dB}$, and then interpolated the resulting values for each and every one of the parameters, i.e., $\eta_{0}, \eta_{1}$ and $\eta_{2}$, in the range of $\sigma_{y} \in[2,6)$ for the UWB systems and in the range of $\sigma_{y} \in[6,12]$ for most of other wireless systems. The results are as follows 


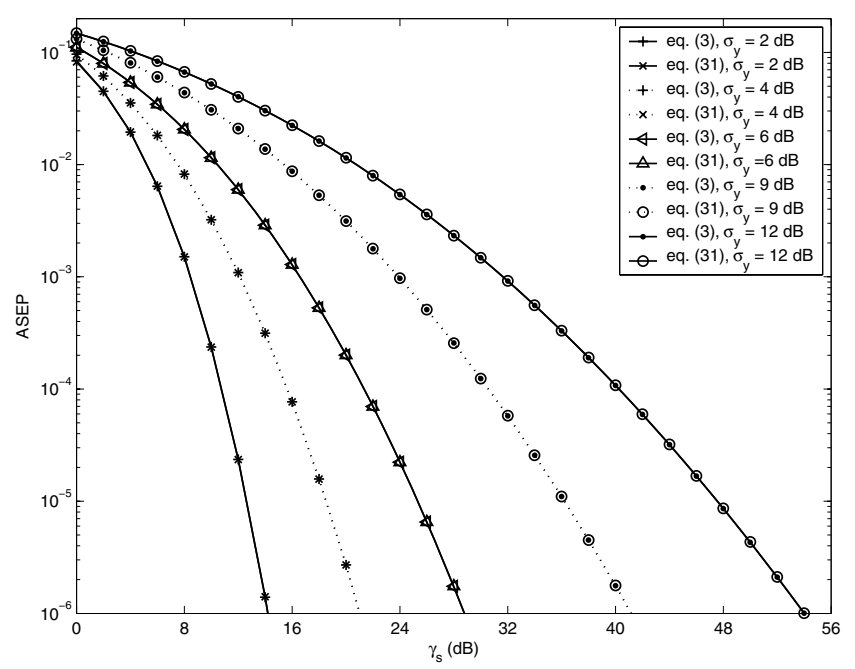

Fig. 3. ASEP vs. SNR per symbol for a binary antipodal constellation and $\sigma_{y}=2,4,6,9,12 \mathrm{~dB}$ using $\overline{P_{e}^{\prime}}$, i.e., eq. (31), and $\overline{P_{e}}$, i.e., eq. (3), relations.

For $\sigma_{y} \in[2,6)$ :

$$
\left\{\begin{array}{l}
\eta_{0}=\left(0.06516\left(\sigma_{y}+24.47\right)\right)^{2}-2.5959 \\
\eta_{1}=-\left(0.06708\left(\sigma_{y}-18.7231\right)\right)^{2}+2.7904 \\
\eta_{2}=\left(0.03162\left(\sigma_{y}-17.001\right)\right)^{2}+0.662
\end{array}\right.
$$

and it implies $\eta_{0} \in[0.379,1.346), \eta_{1} \in[1.532,2.062)$ and $\eta_{2} \in(0.783,0.887]$.

For $\sigma_{y} \in[6,12]$ :

$$
\left\{\begin{array}{l}
\eta_{0}=0.258\left(\sigma_{y}-6\right)+1.345 \\
\eta_{1}=-\left(0.0592\left(\sigma_{y}-21.5\right)\right)^{2}+2.9046 \\
\eta_{2}=\left(0.02646\left(\sigma_{y}-20.07\right)\right)^{2}+0.6434
\end{array}\right.
$$

and it implies $\eta_{0} \in[1.345,2.893), \eta_{1} \in[2.063,2.588)$ and $\eta_{2} \in(0.689,0.782]$. Finally, inserting (29) in (28) and using the values of $\eta_{0}, \eta_{1}$ and $\eta_{2}$ obtained above, we derive a highly tight closed-form approximation of $\overline{P_{e}}$ as follows

$\overline{P_{e}^{\prime}}=\frac{\zeta_{1} \eta_{0}}{\sqrt{2 \pi} \sigma_{\alpha}} \exp \left(-\frac{W_{0}\left(\zeta_{0} \gamma_{s} e^{m_{\alpha}} \sigma_{\alpha}^{2} / 2\right)}{\sigma_{\alpha}^{2}}\right.$

$\left.\times\left[\eta_{1}\left(\frac{W_{0}\left(\zeta_{0} \gamma_{s} e^{m_{\alpha}} \sigma_{\alpha}^{2} / 2\right)}{\sigma_{\alpha}^{2}}\right)^{\eta_{2}-1}+\frac{W_{0}\left(\zeta_{0} \gamma_{s} e^{m_{\alpha}} \sigma_{\alpha}^{2} / 2\right)}{2}\right]\right)$,

(31)

which differs from the APSE $\overline{P_{e}}$ by less than $0.01 \mathrm{~dB}$ for any ordinate values lying between $\overline{P_{e}}=10^{-6}$ and $\overline{P_{e}}=10^{-1}$. In Figures 3, 4 and 5, we plot $\overline{P_{e}}$ in (3) and $\overline{P_{e}^{\prime}}$ in (31) vs. $\gamma_{s}$ $(\mathrm{dB})$ for various $\mathrm{dB}$ spread values and for different types and sizes of constellations. These graphs show a highly tight fitness between $\overline{P_{e}}$ and $\overline{P_{e}^{\prime}}$ for any $\mathrm{dB}$ spread values and constellations presented and, hence, indicate graphically the accuracy of our proposed closed-form approximation in (31). Furthermore, the accuracy of $\overline{P_{e}^{\prime}}$ can be improved for lower $\varepsilon_{0}$ values by a proper choice of the parameters $\eta_{0}, \eta_{1}$ and $\eta_{2}$ in our design formula (30).

\section{ApPlicAtions FOR $\overline{P_{e}^{\prime}}$}

The first application of our closed-form expression is to compute easily and quickly the ASEP of communication

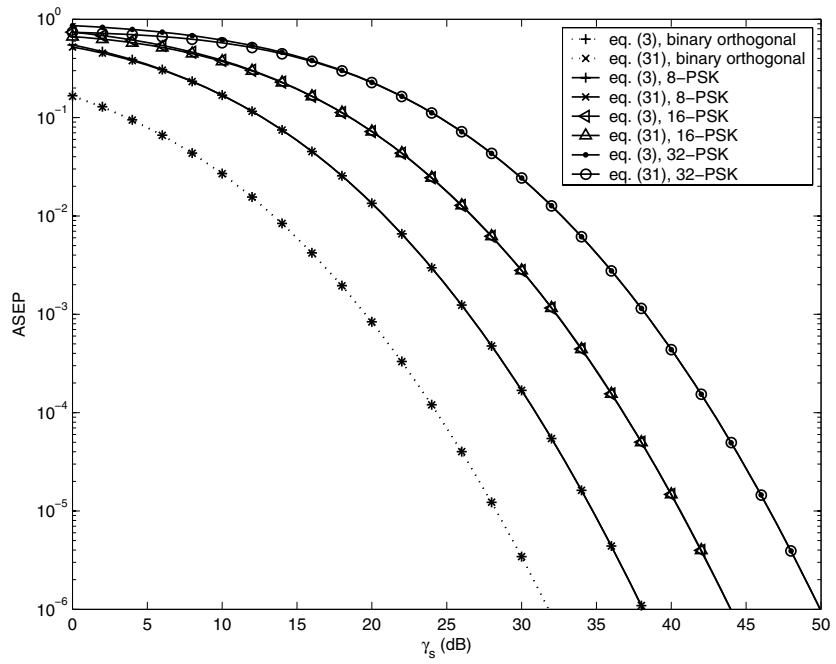

Fig. 4. ASEP vs. SNR per symbol for both binary orthogonal and $M$-PSK constellations and $\sigma_{y}=6 \mathrm{~dB}$ using $\overline{P_{e}^{\prime}}$, i.e., eq. (31), and $\overline{P_{e}}$, i.e., eq. (3), relations.

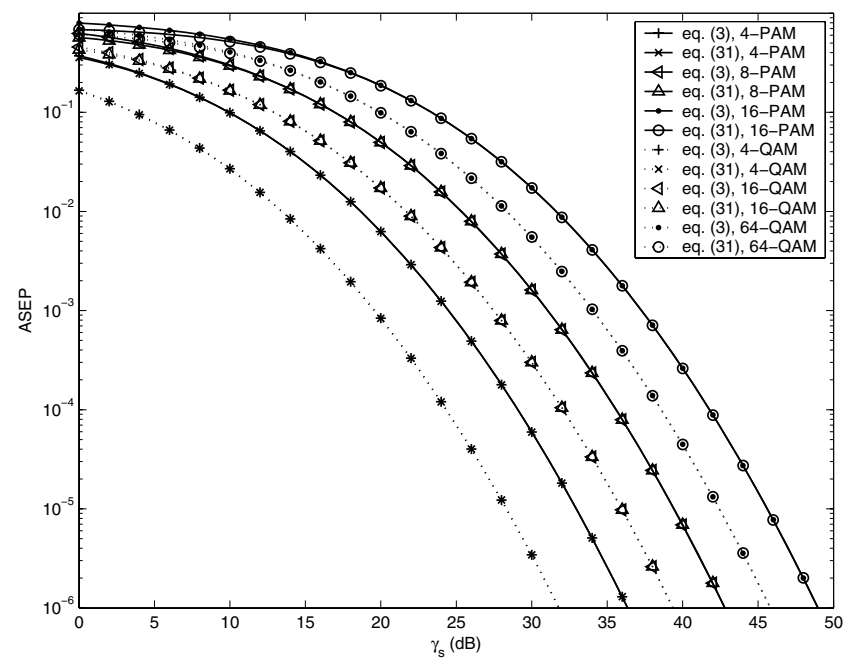

Fig. 5. ASEP vs. SNR per symbol for both $M$-PAM and $M$-QAM constellations and $\sigma_{y}=6 \mathrm{~dB}$ using $\overline{P_{e}^{\prime}}$, i.e., eq. (31), and $\overline{P_{e}}$, i.e., eq. (3), relations.

systems over log-normal fading for different types and sizes of constellations, as shown previously in Figures 3, 4 and 5. Another application of this expression can be found in [11], where it has been applied to establish the theoretical performance of single-link and multiple-antennae single-band UWB systems operating over the IEEE 802.15.3a channel for various modulation schemes, detection methods and types of environments. Also in [11], it has been used to derive new code design criteria, through an upper-bound on the average probability of error, for space-time trellis coding single-band UWB systems operating over the IEEE 802.15.3a channel. Finally, it can be used to get an intuitive understanding of the behaviour of $\overline{P_{e}}$ at high SNRs and to obtain simple parameters to compare the performance of communication systems over log-normal fading. At high SNRs, $\gamma_{s} \gg 1$, the second term of the sum inside the exponential in (31) becomes predominant since $\eta_{2}<0.9$ and the first term can be discarded. Furthermore, knowing that $W_{0}(x \ln (x))=\ln (x)$ 
for $x>1 / e$ [19] and that $x^{d} \geq \ln (x)$ for any $x \geq 0$ and any $d \geq 0.4$ it implies that $W_{0}(x) \geq \ln (x) /(d+1)$ for $x>(1 / e)^{d}$, since $W_{0}$ is monotonically increasing over its domain. Thus, an upper-bound of $\overline{P_{e}^{\prime}}$ can be obtained at high SNRs as

$\overline{P_{e}^{\prime}} \leq \frac{C \zeta_{1} \eta_{0}}{\sqrt{2 \pi} \sigma_{y}}\left[e^{\left(\gamma_{s}(\mathrm{~dB})+\mu\right)^{2}}\right]^{-\frac{1}{2(1+d)^{2} \sigma_{y}^{2}}}=C_{0}\left[e^{\left(\gamma_{s}(\mathrm{~dB})+\mu\right)^{2}}\right]^{-C_{1}}$,

where $\mu=C \ln \left(\zeta_{0} e^{m_{\alpha}} \sigma_{y}^{2} / 2 C^{2}\right)$ and $C_{0}$ and $C_{1}$ are positive constants. In comparison, assuming a transmission of data over a Rayleigh or a Rician fading channel, the ASEP at high SNRs can be formulated as follows

$$
\overline{P_{e}} \leq D_{0}\left[e^{\left(\gamma_{s}(\mathrm{~dB})\right)}\right]^{-D_{1}}
$$

where $D_{0}$ and $D_{1}$ are positive constants. Both of these bounds in (32) and (33) are loose bounds that describe the asymptotic behaviour of the ASEP at high SNRs. It is well-known that over a Rayleigh fading channel, the ASEP at high SNRs is a linear function of the $\mathrm{SNR}(\mathrm{dB})$ considering a semi-log scale [1], as it is clearly proven by (33). Similarly, (32) shows that the ASEP at high SNRs over a log-normal fading channel is a quadratic function of the $\mathrm{SNR}(\mathrm{dB})$ considering a semi-log scale.

\section{CONCLUSION}

An accurate closed-form approximation of the ASEP over a log-normal fading channel has been derived for any practical $\mathrm{dB}$ spread values and various constellation sizes and types. First, using the approximation of $Q(x)$ in [16] and a simple linear approximation for $f(x)$, we have obtained an expression $\widetilde{\overline{P_{e}}}$ that closely approximates $\overline{P_{e}}$ for large values of $I, L$, and $N$. However, we have noticed that even for small values of these parameters, the slopes of $\overline{P_{e}}$ and $\overline{P_{e}}$ presented parallel features at high SNRs. Then, using this intuition, we have modified the simplest $\widetilde{\bar{P}_{e}}$ expression by introducing a parametric function and a criterion with an adjustable accuracy to obtain our accurate approximation, i.e., $\overline{P_{e}^{\prime}}$, for $\overline{P_{e}}$. The great accuracy of $\overline{P_{e}^{\prime}}$ has been obtained for a typical range of average error probability in mobile communications by optimizing the values $\eta_{0}, \eta_{1}$ and $\eta_{2}$ in our design formula, and has been shown experimentally for practical $\mathrm{dB}$ spread values and various constellation sizes and types. Some applications of our closed-form approximation have also been presented, for instance to establish the theoretical performance of singleband UWB systems or to evaluate and compare quickly the symbol-error performance of communication systems over log-normal fading channels. Finally, an upper bound of $\overline{P_{e}^{\prime}}$ has been found that provides an intuitive understanding of the behaviour of $\overline{P_{e}}$ at high SNRs.

\section{REFERENCES}

[1] J. Proakis, Digital Communications, 4th ed. McGraw-Hill, 2001.

[2] M. K. Simon and M.-S. Alouini, Digital Communication over Fading Channels: A Unified Approach to Performance Analysis. Wiley, 2000.

[3] Q. Zhang and T. Tjhung, "Outage performance of cellular systems over arbitrary lognormal shadowed rician channels," IEE Electron. Lett., vol. 35, no. 15, pp. 1227-1229, July 1999.

[4] J. Foerster et al., "Channel modelling sub-committee report final," IEEE P802.15 Wireless Personal Area Networks, Tech. Rep. P802.1502/490r1-SG3a, Feb. 2003.

[5] J. Foerster and Q. Li, "UWB channel modelling contribution from intel," Intel, Tech. Rep. P802.15-02/490r1-SG3a, 2002.

[6] D. Cassioli, M. Z. Win, and A. F. Molisch, "The ultra-wide bandwidth indoor channel: from statistical model to simulations," vol. 20, no. 6 , pp. 1247-1257, Aug. 2002.

[7] M. Ghavami, L. Michael, and R. Kohno, Ultra Wideband Signals and Systems in Communication Engineering. Wiley Europe, May 2004.

[8] A. A. M. Saleh and R. A. Valenzuela, "A statistical model for indoor multipath propagation," IEEE J. Select. Areas Commun., vol. 5, no. 2, pp. 128-137, Feb. 1987.

[9] M. Abramowitz and I. A. Stegun, Handbook of Mathematical Functions with formulas, Graphs and Mathematical Tables. New-York: Dover Press, 1974.

[10] R. M. Corless, G. H. Gonnet, D. E. G. Hare, D. J. Jeffrey, and D. E. Knuth, "On the lambert W function," Adv. Comput. Math., vol. 5, pp. 329359, 1996.

[11] F. Héliot, "Design and analysis of space-time block and trellis coding schemes for single-band UWB communications systems," Ph.D. dissertation, King's College London, University of London, July 2006. [Online]. Available: http://membres.lycos.fr/fheliot/pub/PhD_thesis.pdf

[12] J. Gubner and K. Hao, "An exact computable formula for the average bit-error probability of the IEEE 802.15.3a UWB channel model," in Proc. IEEE ICUW 2005, Zurich, Switzerland, Sept. 2005, pp. 142-146.

[13] H. Liu, "Error performance of a pulse amplitude and position modulated ultra-wideband system over lognormal fading channel," IEEE Commun. Lett., vol. 7, no. 11, pp. 531-533, Nov. 2003.

[14] A. J. Goldsmith and S.-G. Chua, "Adaptive coded modulation for fading channels," IEEE Trans. Commun., vol. 46, no. 5, pp. 595-602, May 1998.

[15] N. C. Beaulieu, A. A. Abu-Dayya, and P. J. Mclane, "Estimating the distribution of a sum of independent lognormal random variables," IEEE Trans. Commun., vol. 43, no. 12, pp. 2869-2873, Dec. 1995.

[16] M. Chiani, D. Dardari, and M. K. Simon, "New exponential bounds and approximations for the computation of error probability in fading channels," IEEE Trans. Wireless Commun., vol. 2, no. 4, pp. 840-845, 2003.

[17] F. Héliot, M. Ghavami, and M. R. Nakhai, "An accurate closed-form approximation of the average probability of error over a log-normal fading channel : Derivation details," Tech. Rep., Sept. 2007. [Online]. Available: http://membres.lycos.fr/fheliot/pub/Tech_note_1.pdf

[18] A. Jeffrey, Mathematics for Engineers and Scientists. Chapman \& Hall, Aug. 2004

[19] "The wolfram research site." [Online]. Available: http://functions. wolfram.com/ElementaryFunctions/ProductLog/16/01/ 